\title{
Early Inflammatory Arthritis
}

National Cancer Institute

\section{Source}

National Cancer Institute. Early Inflammatory Arthritis. NCI Thesaurus. Code C119022.

Early stages of inflammatory arthritis, when the underlying diagnosis is uncertain, usually little or no joint damage has occurred. Treatment may alter the disease course (e.g., potentially avert progression to full RA or other diagnosis). 